\title{
Comportamiento Clínico de la Leihsmaniosis Visceral en Población Infantil. Diez Años de Experiencia de un Centro de Referencia del Paraguay
}

\section{Clinical behavior of Visceral Leishmaniasis in children. Ten Years of Experience Reference Center Paraguay}

Dr. Silvio Apodaca, Dra. Soraya Araya, Dra. Celia Martínez de Cuellar, Dra. Dolores Lovera, Prof. Dr. Antonio Arbo. Servicio de Pediatría, Instituto de Medicina Tropical

Objetivo: Analizar las características clínicas, laboratoriales y evolución de pacientes pediátricos hospitalizados por leishmaniosis visceral en el Instituto de Medicina Tropical. Población y métodos: Estudio observacional realizado en el Instituto de Medicina Tropical en pacientes <15 años hospitalizados durante el periodo 2003 al 2013 con diagnóstico de leishmaniosis visceral. El diagnóstico de leishmaniosis visceral se realizó por la visualización de amastigotes en la médula ósea y/o positividad del Rk 39. Resultados: Durante el periodo de estudio 53 pacientes fueron identificados, con una edad media 35,4 pts \pm 32 meses, (relación Femenino / Masculino: 28/27). La mayoría de los casos se encontraron en el grupo de edad < 2 años 28/53 (52 \%), seguido por el grupo de 2 a 5 años. El tiempo medio entre la aparición de síntomas y la hospitalización fue de $73 \pm 43$ días y el promedio de días de internación $22 \pm 10$ días. La tríada de fiebre, hepatoesplenomegalia estuvo presente en prácticamente todos los pacientes. Anemia, leucopenia y trombocitopenia en $94 \%, 58 \%, 58 \%$ de los casos, respectivamente $y$ pancitopenia en el $38 \%$ de los pacientes. Se constató amastigotes en médula ósea en el $94 \%$ (50/53) de los pacientes, serología positiva para RK39 en $51 / 53$ pacientes (96\%). Se observaron complicaciones en $33 / 53$ pacientes (62\%), en su mayoría infecciosas, de las cuales (32\%) presentaron neumonía y 9 (17\%) infección del tracto urinario. Siete pacientes (13\%) presentaron síndrome hemofagocítico. En cuanto al tratamiento inicial 47 pacientes (91\%) fueron tratados con compuestos antimoniales, de los cuales 2 pacientes precisaron cambio de tratamiento a anfotericina $B$, por efectos adversos de la medicación. 6 pacientes (9\%) recibieron tratamiento inicial con anfotericina B liposomal (esquema clásico: 5/6, tratamiento acortado con monodosis de $10 \mathrm{mg} / \mathrm{k} / \mathrm{d} ; \mathrm{1} / 6$ ), todos con síndrome Hemofagocítico asociado. Solo 1 paciente presentó recaída de la enfermedad. La mortalidad registrada fue del 2\% (1/53).

Palabras claves: hepatomegalia, Instituto de Medicina Tropical, niños 


\section{Abstract}

Aim: To analyze the clinical, laboratory and development of pediatric patients hospitalized for visceral leishmaniasis at the Institute of Tropical Medicine features.

Population and methods: observational study conducted at the Institute of Tropical Medicine in patients $<15$ years hospitalized during the period 2003 to 2013 with diagnosis of visceral leishmaniasis. The diagnosis of visceral leishmaniasis was performed by visualizing amastigotes in bone marrow and / or positivity rK 39.

Results: During the study 53 patients were identified, mean age $35.4 \pm 32$ pts months (ratio Female / Male: 28/27). Most cases were in the age group $<2$ years $28 / 53$ (52\%), followed by the group of 2-5 years. The mean time between onset of symptoms and hospitalization was $73 \pm 43$ days and the average days of hospitalization $22 \pm 10$ days. The triad of fever, hepato-splenomegaly was present in almost all patients. Anemia, leukopenia and thrombocytopenia in 94\%, 58\%, 58\% of cases, respectively, and pancytopenia in $38 \%$ of patients. It was found amastigotes in bone marrow in 94\% (50/53) of patients seropositive for rK39 in 51/53 patients (96\%). Complications in 33/53 patients (62\%), most infectious, of which (32\%) had pneumonia and 9 (17\%) urinary tract infection were observed. Seven patients (13\%) had hemophagocytic syndrome. As for the initial treatment 47 patients (91\%) were treated with antimonial compounds, of which 2 patients required treatment change to amphotericin $B$, due to adverse effects of medication. 6 patients (9\%) received initial treatment with liposomal amphotericin B (classical scheme: 5/6, shortened treatment with single doses of 10 mg/kg/d: 1/6), all associated hemophagocytic syndrome. Only one patient had recurrence of the disease. Registered mortality was 2\% (1/53)

Key words: children, Institute of Tropical Medicine, hepatosplenomegaly.

\section{Introducción}

La Leishmaniosis visceral es una zoonosis con una variedad de huéspedes reservorios mamíferos, incluidos perros y roedores.

A nivel mundial, más de 350 millones de personas se encuentran en riesgo de padecer la enfermedad, siendo 88 los países que han reportado casos de leishmaniosis visceral de los cuales 72 son países en vías de desarrollo (1)

El número anual estimado de casos nuevos en el mundo es de 500.000, correspondiendo el $90 \%$ de los casos reportados a India, Bangladesh, Sudán y Brasil (2)

Históricamente el primer caso americano fue descubierto por Migone en 1911, en Paraguay (2). Desde esa fecha hasta los últimos años del siglo XX han sido encontrados unos pocos casos esporádicos, sin embargo actualmente el número de casos registrados de Leishmaniosis visceral aguda (LVA) ha aumentado en forma notable en los últimos años, en Paraguay. En el año 2000 se registró solamente un paciente con esta patología, en el año 2002 se registraron 4 casos, en el 2003 fueron 9casos, en el año 2004 fueron 24 casos, creciendo en forma constante, hasta registrarse 82 casos en el 2009 incluyendo los casos en población adulta (2).

En nuestro país más del $90 \%$ de los casos de Leishmaniosis visceral provienen 
del área geográfica que abarca al distrito de Asunción y a los departamentos de Central, Paraguarí y Cordillera, probablemente debido a la instalación del ciclo de transmisión de la enfermedad, en donde convergen una alta proporción de perros con LVA y flebótomos vectores, además de un crecimiento urbano desordenado de la población.

En menor proporción también se han detectado casos en los departamentos del Guairá, Itapúa, Misiones, Alto Paraná, Concepción y Amambay.

El objetivo del presente estudio ha sido la caracterización clínica- epidemiológica de la leishmaniosis visceral en población pediátrica y respuesta al tratamiento antimonial.

\section{Materiales y métodos}

Estudio retrospectivo realizado en el Instituto de Medicina Tropical de Asunción Paraguay, y está basado en la revisión de las historias clínicas de pacientes internados en el Servicio de Pediatría, con diagnóstico de alta de Leishmaniosis Visceral.

El Instituto de Medicina Tropical constituye el principal centro de referencia para internación de pacientes con patologías tropicales, procedentes de las áreas geográficas de Asunción y Gran Asunción, la cual incluye a la capital del país con 750.000 habitantes y ciudades más pequeñas que rodean a la capital, para una población total de 1.200 .000 habitantes. El hospital admite 1.000 pacientes menores 15 años de edad por año.

En la presente serie se incluyeron pacientes menores de 15 años de edad con diagnóstico de Leishmaniasis visceral hospitalizados entre enero de 2003 y abril de 2013.

La leishmaniasis visceral se definió como la identificación de la forma amastigote de Leishmania $s p$. en frotis de médula ósea, prueba serológica positiva (prueba basada en rK39 inmunocromatográfico o ELISA), o ambos, en un paciente con manifestaciones compatibles con leishmaniasis visceral (es decir, fiebre, hepatoesplenomegalia, anemia, leucopenia y trombocitopenia).

Se analizaron todos los registros médicos, utilizando una proforma de protocolo estructurado para la recopilación de datos epidemiológicos (edad, sexo, lugar de residencia, condiciones de vivienda), datos de laboratorio clínico, duración de los signos y síntomas, el examen físico, pruebas de diagnóstico, datos de laboratorio, tratamiento y evolución.

\section{Definición de Variables}

- Fiebre: Temperatura axilar $>38^{\circ} \mathrm{C}$.

- Anemia: Valor de hemoglobina $<10$ g/dl

- Leucopenia: Recuento de Glóbulos blancos menor a $4000 \mathrm{~mL}$

- Trombocitopenia: Recuento de plaquetas inferior a 150.000 plaquetas / $\mathrm{ml}$

Criterios diagnósticos para Síndrome hemofagocítico:

Presencia de:

- Fiebre: Picos de $38,5^{\circ} \mathrm{C}$, mínimo 7 días,

- Esplenomegalia de $3 \mathrm{~cm}$ y,

- Una de dos de los siguientes:

- Anemia < de 9 de Hb,

- Trombocitopenia < 100.000 . 
- Neutropenia < 1.000 .

- Hipertrigliceridemia $>2,0 \mathrm{mmol} / \mathrm{L}$ $0>3$ DS o

- Hipofibrinogenemia $<1,5 \mathrm{gr} / \mathrm{L} 0<$

3 DS (< de 150),

- Hemofagocitosis sin evidencia de MO hipoplásica.

Se consideró desnutrición cuando el score $Z$ (peso/edad) fue menor a 2 desvíos estándar.

Se evaluaron las características clínicopatológicas, con énfasis en las complicaciones y el rendimiento diagnóstico de la citología de médula ósea en comparación con la serología RK39.

Para el análisis de datos se recurrió al programa EpiInfo $₫$ para Windows ${ }^{\circledR}$ 3.4.3 Centers for Disease Control and Prevention, Atlanta, Georgia, United States of America). Para la comparación de proporciones se utilizó $X^{2}$.
Se consideró estadísticamente significativo un valor $p<0,05$.

\section{Resultados}

De enero de 2003 a abril de 2013, se diagnosticaron un total de 53 casos de leishmaniosis visceral.

La edad media fue de 35,4 \pm 32 meses, y la distribución por sexo fue similar (relación Femenino / Masculino: 28 / 27) Ningún paciente VIH fue positivo.

La mayoría de los casos se encontraron en el grupo de edad < 2 años 28 / 53 (52 $\%)$, seguido por el grupo de 2 a 5 años (Tabla 1). El análisis de la situación nutricional de los pacientes mostró que 7 pacientes $(13 \%)$ estaban por debajo del percentil 10 en las tablas de crecimiento de talla para la edad.

Tabla 1- Características Clínicas de los pacientes con Leishmaniasis Visceral

\begin{tabular}{lcc}
\hline \multicolumn{1}{c}{ Variables } & $\mathbf{n}$ & \% \\
\hline Edad media & $35.4 \pm 32$ & \\
Femenino / Masculino & $28 / 27$ & $1.03: 1$ \\
Edad & 28 & \\
$\quad<2$ años & 13 & 52 \\
$2-5$ años & 12 & 25 \\
$\quad 5$ años & $22 \pm 10$ & 23 \\
Promedio días de Internación & 33 & 62 \\
Complicaciones & 5 & 9 \\
Ingreso a UCIP & 1 & 2 \\
Óbitos & & \\
\hline
\end{tabular}

El tiempo medio entre la aparición de síntomas y la hospitalización fue de $73+43$ días. El promedio de días de internación fue $22 \pm 10$ días.
Las características clínicas y de laboratorio relevantes se muestran en la tabla 2. 
Tabla 2- Hallazgos clínicos y laboratoriales de los pacientes con Leishmaniosis visceral

\begin{tabular}{lc}
\hline Signos & $\mathbf{n = 5 3 ( \% )}$ \\
Fiebre & $52(98)$ \\
Esplenomegalia & $53(100)$ \\
Hepatomegalia & $50(94)$ \\
\hline Hallazgos Laboratoriales & \\
Anemia & $50(94)$ \\
Leucopenia & $29(55)$ \\
Trombocitopenia & $31(58)$ \\
Pancitopenia & $20(38)$ \\
Enzimas Hepáticas $>3$ veces el valor normal & $17(32)$ \\
\hline
\end{tabular}

La tríada de fiebre, hepatomegalia y esplenomegalia estuvo presente en prácticamente todos los pacientes. Casi todos los pacientes fueron ingresados con anemia 50 / 53 (94\%). La leucopenia y la trombocitopenia se observaron en una proporción sustancial de los pacientes 31 / 53 (58\%).

Se constató pancitopenia en el $38 \%$ de los pacientes y un tercio de la serie $17 / 53$ (32 \%) presentó al ingreso niveles elevados de enzimas hepáticas (3 veces superiores al valor normal)
El diagnóstico de leishmaniosis visceral se basó en la observación de amastigotes en la médula ósea, la cual fue positiva en el $94 \%$ (50 / 53) de los pacientes y la serología RK39 fue positiva en 51/53 (96\%)

Se observaron complicaciones en $33 / 53$ pacientes $(62 \%)$, en su mayoría infecciosas (neumonía, infección urinaria, piodermitis, gastroenteritis). Diecisiete pacientes (32\%) presentaron neumonía y $9(17 \%)$ infección del tracto urinario. Siete pacientes $(13 \%)$ presentaron manifestaciones clínicas y laboratoriales compatibles con síndrome hemofagocítico (tabla 3).

\section{Tabla 3- Complicaciones en pacientes con Leishmaniasis Visceral}

\begin{tabular}{lcc}
\hline \multicolumn{1}{c}{ Complicaciones } & $\mathbf{n = 5 3}$ & $\mathbf{\%}$ \\
\hline Infección Urinaria & 9 & 17 \\
Neumonía & 17 & 32 \\
Otras infecciones & 9 & 17 \\
Sindrome Hemofagocítico & 7 & 13 \\
\hline
\end{tabular}

En cuanto al tratamiento inicial 47 pts (91\%) fueron tratados con compuestos antimoniales (Estibogluconato de sodio a 20 $\mathrm{mg} / \mathrm{k} /$ día por 28 días). De este grupo 2 pacientes precisaron cambio de tratamiento a anfotericina $B$, por efectos adversos de la medicación. 6 pacientes $(9 \%)$ recibieron tratamiento inicial con anfotericina $B$ liposomal (esquema clásico: 5/6, tratamiento acortado con monodosis de 10 $\mathrm{mg} / \mathrm{k} / \mathrm{d}$ : $1 / 6$ ) todos ellos con síndrome Hemofagocítico asociado. 
Del total de pacientes hospitalizados, antimoniales, presentó recaída de la uno solo que había recibido tratamiento con enfermedad (tabla 4).

Tabla 4- Tratamiento de los pacientes con leishmaniasis visceral

\begin{tabular}{lcc}
\hline \multicolumn{1}{c}{ Tratamiento } & $\mathbf{n = 5 3}$ & \% \\
\hline Antimoniales & 47 & 89 \\
Anfotericina B & 6 & 11 \\
Recambio a Anfotericina B & 2 & 4 \\
Recaída & 1 & 2 \\
\hline
\end{tabular}

La mortalidad registrada fue $2 \%(1 / 53)$, correspondiendo al paciente que recibió tratamiento acortado

\section{Discusión y Conclusión}

En el presente estudio destaca la importancia de la leishmaniosis visceral en nuestro país, la mayor frecuencia e incidencia de la leishmaniosis visceral en la edad pediátrica radica en la inmadurez del estado inmunológico.

La edad media fue de 35,4 \pm 32 meses, y la distribución por sexo fue similar, ningún paciente VIH positivo.

La mayoría de los casos se encontraron en el grupo de edad $<2$ años.

La tríada de fiebre, hepatomegalia y esplenomegalia (3) estuvo presente en prácticamente todos los pacientes. Casi todos los pacientes fueron ingresados con anemia (94\%). La leucopenia y la trombocitopenia se observaron en una proporción sustancial de los pacientes (58\%), pancitopenia $38 \%$

Las complicaciones infecciosas (4) fueron comunes, sobre todo neumonía e infección del tracto urinario.
Frecuencia no despreciable (13\%) de síndrome de activación macrofágica relacionada a leishmaniosis visceral.

El pronóstico de la leishmaniasis visceral en niños inmunocompetentes, con tratamiento oportuno por lo general es bueno.

En población pediátrica del Paraguay, el fracaso de la terapia con compuestos antimoniales fue excepcional por lo cual en el Instituto de Medicina Tropical, Departamento de Pediatría, hemos adoptado como protocolo de tratamiento el uso de fármacos antimoniales, reservando la Anfotericina B (desoxicolato o liposomal) para circunstancias especiales tales como: reacción adversa al estibogluconato de sodio o meglumina, pacientes con leishmaniosis visceral complicada con Síndrome Hemofagocítico y /o comorbilidad por VIH (5)

\section{Referencias}

1. Barral A, Badaró R, Barral-Netto $M$, et al. Isolation Leishmaniamexicanaamazonensis from the bonemarrow in a case of American visceral leishmaniasis. Am J Trop Med Hyg 1986; 35:732.

2. Monroy-Ostria A, Hernandez-Montes O, Barker DC. Aetiology of visceral 
leishmaniasis in Mexico.Acta Trop2000; 75:155.

3. Sacks DL, Kenney RT, Kreutzer RD, et al. Fecha de recepción: 06 de octubre de 2014 Indian kala-azar caused by Leishmaniatropica. Lancet Fecha de aceptación: 12 de febrero de 2015 1995;345:959.

4. Bogdan C. Mechanisms and consequences of persistence of intracellular pathogens: leishmaniasis as Solicitud de Sobretiros: anexample. Cell Microbiol 2008; 10:1221. Prof. Dr. Antonio Arbo Instituto de Medicina Tropical

5. Bogdan C, Röllinghoff M. The immune antonioarbo@hotmail.com response to Leishmania: mechanisms of parasite control and evasion. Int J Parasitol 1998; 28:121. 